\title{
Analysis and simulation of three MANET routing protocols: A research on AODV, DSR \& DSDV characteristics and their performance evaluation
}

\author{
Murad Ghazy Khalaf Alabdullah ${ }^{1}$, Bassam Mohsin Atiyah $^{2}$, Kaesar Sabah Khalaf ${ }^{3}$, Saber Hameed \\ Yadgar $^{4}$ \\ ${ }^{1}$ Department of Electronic Techniques, Kirkuk Technical Institute, Northern Technical University, Iraq \\ ${ }^{2}$ Department of Electronic Techniques, Kirkuk Technical Institute, Northern Technical University, Iraq \\ ${ }^{3}$ Department of Electronic and Control Engineering, Kirkuk Technical College, Northern Technical University, Iraq \\ ${ }^{4}$ Department of Electronic Techniques, Kirkuk Technical Institute, Northern Technical University, Iraq
}

\section{Article Info}

Received Jun 05, 2019

\begin{abstract}
Mobile ad-hoc network allows electronic devices to independently configure the communication; even if the device changes its position, the (MANET) can maintain the connectivity among the devices since it has the capability to arrange a temporary network dynamically. The lack of central infrastructure and the freedom of mobile nodes to move randomly will create several problems, like routing and security issues. MANET requires a stable routing algorithm to adapt to the network that changes its topology randomly at any time. Several studies were conducted with different scenarios to suggest the best protocol for routing. However, a clear performance evaluation is still a missing part, because the dissimilarities in the mechanism of the protocol can lead to important performance differences. This article provides a performance assessment of (DSR), (DSDV), and (AODV) protocols. Research results and the evaluation of the network are made based on network size, mobility, and variable network load by using NS2 application for simulation. The results reveal that the (AODV, DSR) are more efficient than DSDV and reasonably more proper for ad-hoc applications and projects.
\end{abstract}

Keyword:

MANET

Routing Protocols

DSR

AODV

DSDV

\section{Corresponding Author:}

Murad Ghazy Khalaf Alabdullah

Departement of Electronic Techniques

Northern Technical University

Kirkuk Technical Institute, Kirkuk, Iraq

Email: murad.kerkukly@gmail.com 


\section{Introduction}

The new technology has made wireless communication to be an evolving and emerging technology that will provide consumers with electronic access to data and services wherever they are [1]. These specifications will be met by the wireless connection to the well-Known local area network. However, in situations or places where there's no available base station or fixed infrastructure, there is a growing demand for connectivity or being online. These requirements are solved by the ad-hoc network [2], [4], [20], [21]. MANET's networks are autonomously organized and Self-configured without any infrastructure assistance and it can be installed quickly and easily at a very low cost [3], [5], and [9]. Since node mobility is high, unpredictable topology modifications may occur in such networks. Mobility and the lack of existing infrastructure make MANETs very appealing to time-critical apps. There is a need for a routing algorithm when a packet or data wants to be transferred to a node through a set of different nodes. A routing protocol is a fundamental requirement for the network to create routing decisions even for the immobile network. All of the network nodes function like routers and play a role in maintaining routes to other network nodes. In the case of emergency or search and rescue operations, ad-hoc networks are very useful for gathering information in difficult terrains or in meetings or conferences where people want their data to be shared fast. Routing is a network layer task which decides the path between the source and the target for traffic flow. It can be seen at first glance that directing in wireless (multi-hop) network causes no other issue than dealing with a very dynamic topology [12].

Dynamic wireless networks reveal that traditional routing algorithms for wired networks are either unstable or require a large number of protocol loads for routing updates. The topology change needs to be responded quickly because the delivery of the packages to the relevant locations is not possible until the topology is stable. MANETs ' limited resources have given it a hard challenge to design a robust routing strategy. The use of these limited resources requires an effective routing algorithm, at the same time being dynamic to any variations in network circumstances like the size of the network, traffic density, and mobility of nodes, topology, and even broken routes [3], [23], [24]. Due to these issues, new algorithms for routing are proposed in MANETs. Routing protocol should have the capability to determine the best direction, less overhead and power consumption reduces the time needed to converge and maximize the use of bandwidth after topology modifications. In this study, we compare the network efficiency according to packet delivery fraction, packet latency average to evaluate performance traffic and normalized load for routing, by changing the number of sources, velocity and break time of nodes.

\section{Related Works}

Many attempts by researchers are made to find the optimum protocol that can be applied for a defined network and they employed different scenarios to catch the best results, this work is also evolving the prior research in this field. Rohit Kumar [14] has made a study between reactive and proactive routing protocols with different mobility models, the results show that all protocols present good results when the nodes are fixed or moves less, but when there is a rise in node mobility and dynamic change in network topology, then the best protocols are FSR and AODV. A. K. Dwivedi et al [2] have studied the routing protocols performance with various node mobility models, two protocols were examined which are DSR and AODV with different speed of node mobility, they showed that DSR performs well with low traffic and low mobility, while AODV shows better performance when the mobility and the traffic is high. Charu Wahi et al [3] have made a comparative study on DSDV, AODV, and DSR, they showed advantage and limitations of every protocol, the DSDV has high overhead but it is loop-free, AODV has large delays and scalability problems and DSR displays scalability difficulties because of flooding and source routing but it can send the packet with multiple routes, therefore, A single routing protocol cannot perform well in all scenarios and they have suggested that protocol selection should be made in accordance with necessities of the application. Sachin Dnyandeo Ubarhande [4] investigated AODV and DSR using (NS2) software to conduct a comparative study with 
different metrics. Research showed that DSR showed pretty good results in terms of packet loss, while AODV presents better performance than DSR when there is a huge TCP packet sending rate attempt. Charu Wahi and Sanjay Kumar Sonbhadra [5] made a comparative study about routing protocols, a classification of protocols categories have been presented, they found it so hard to determine which protocol would best perform under a variety of distinct MANET models.

Diaa Eldein and Othman O. Khalifa [10] have worked on a comprehensive classification about all available ad-hoc protocols. Furthermore, a detailed comparison has been produced between some cases of each kind of routing protocols to further support research in this field.

\section{Problem Definition}

The aim of the research is to show suggested DSDV, DSR, and AODV protocols capability with different metrics and conditions. The research also seeks to produce a simulation environment within the range of the design-free network region that can be used as a basis for future studies. This simulation setting is focused on Berkeley's Network Simulator 2. Efficiency will be analyzed by considering the following metrics; (Packet delivery fraction), (Average packet latency), and (Normalized routing load). The main goal is to reach the best performing protocol which will be suitable for the presented scenario under various metrics.

\section{Classification of Routing Protocols and Design Challenges}

The classification, in general, can be divided into two parts [15], [10]:

1. Routing Strategy.

2. Network Structure.

If we consider (Network Structure) we see it is also classified into five parts as below [10]:

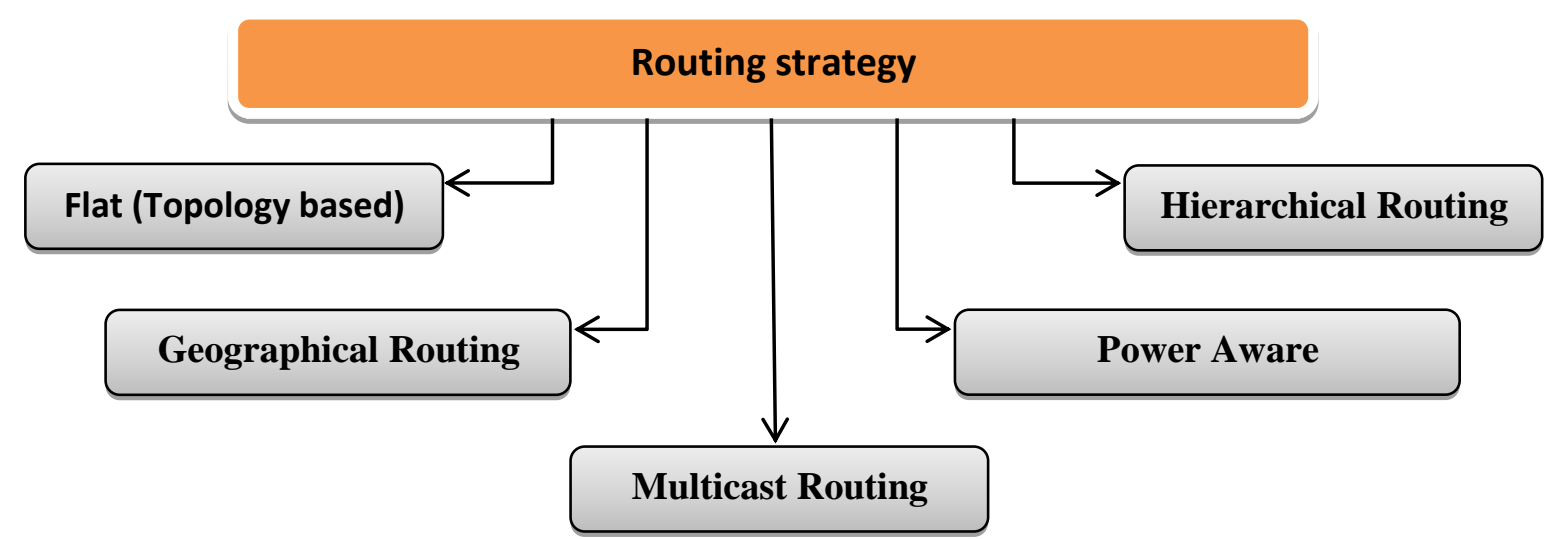

Figure 1. Network structure classification [10]

Our study puts emphasis on the first one; topology based also can be categorized as follow:

- Proactive - table based processing protocols [7], [8], [9], [10].

- Reactive - demand based routing protocols [7], [8], [9], [10].

- Hybrid - possess both (proactive and reactive) protocols features [8], [9], [10].

The classification of the existing routing protocols types is as follow: 


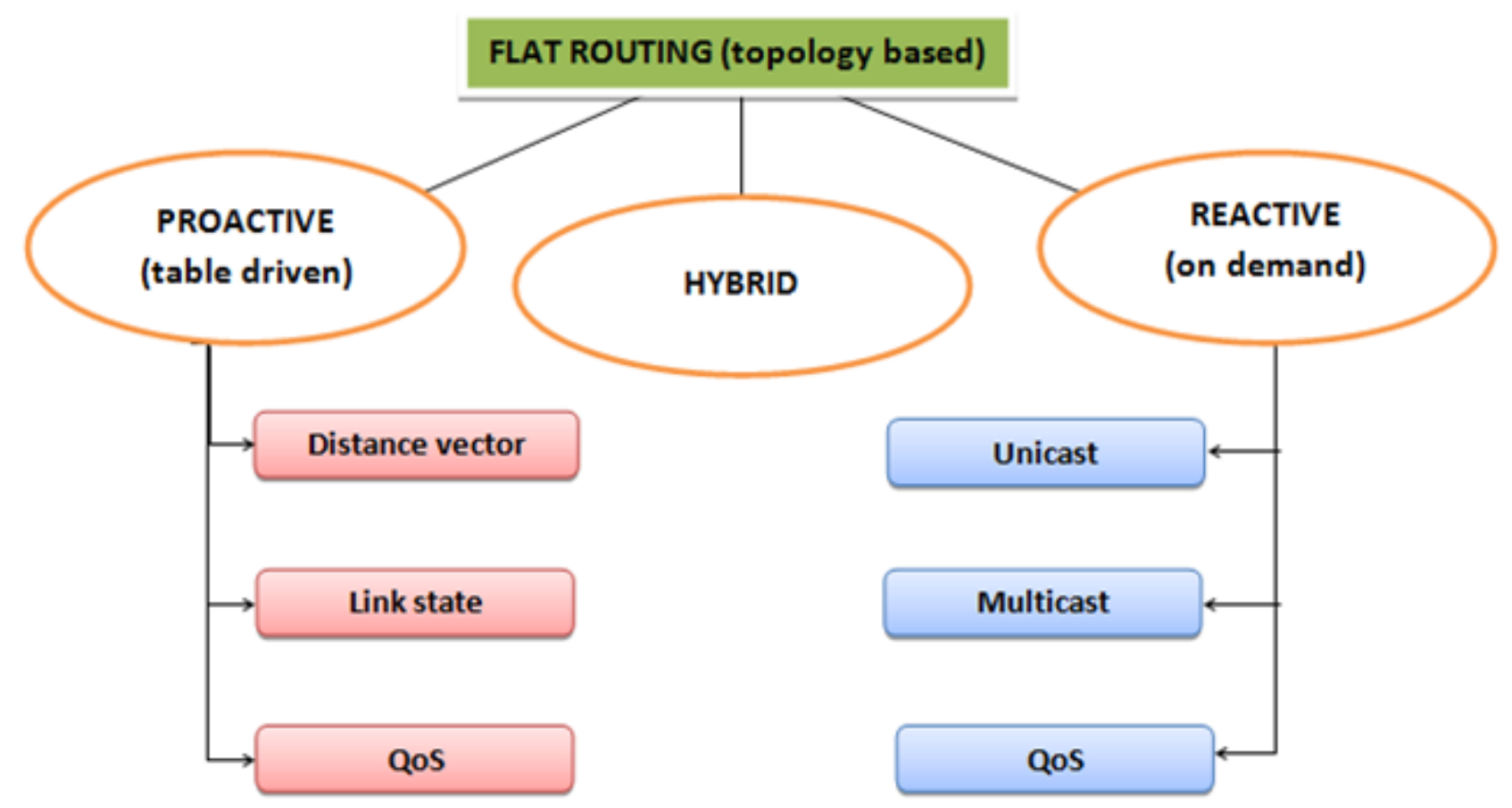

Figure 2. Available routing protocol classification [10]

Every mentioned subdivision in the classification has several protocols, in our article we took the following protocols as a study case; DSDV is a distance vector protocol, whereas DSR and AODV are demand based unicast protocol.

There are numerous other protocols which might be a good chance for future work. For instance, these kinds of protocols can be organized into three components as below:

Table 1. Driven Protocols (Proactive)

\begin{tabular}{|c|c|c|}
\hline 1. Distance Vector & 2. Link State & 3. QoS \\
\hline DSDV & OLSR & CEDAR \\
\hline CGSR & OSPF & QOLSR \\
\hline RIP & FSR & \\
\hline LRR & STAR & \\
\hline WRP & GSR & \\
\hline
\end{tabular}

Table 2. On-Demand routing protocols (Reactive)

\begin{tabular}{|c|c|c|}
\hline 1. Unicast & 2. Multicast & 3. $\boldsymbol{Q o s}$ \\
\hline OSPF & TORA & MP-DSR \\
\hline DSR & AOMDV & ODLW \\
\hline AODV & DMSR & AQOR \\
\hline DYMO & CHAMP & \\
\hline
\end{tabular}


Table 3. Hybrid Protocols

\begin{tabular}{|c|}
\hline Hybrid \\
\hline ZRP \\
\hline LANMAR \\
\hline RDMAR \\
\hline HOPNET \\
\hline DDR \\
\hline
\end{tabular}

\section{Characteristics of Protocols}

\subsection{AODV Protocol Definition}

Algorithm of the routing for the Demand-Based Distance Vector (AODV) is a request-based algorithm, that is, it only creates paths between nodes if the source nodes need it, and these routes are only kept as long as they are needed [5], [6]. In AODV, a sequence number is informed about any change in the neighboring topology. This sequence number allows the selection of the most current route each time a route is searched. AODV uses connections between adjacent nodes. Thus, to save the corresponding routing information, both unicast, multicast routing tables are used. These tables can keep both, even for fixed nodes. Moreover, AODV reacts to modifications in the topology that rapidly impact active paths. It builds routes with only a few amounts of routing control messages and without any extra network protocol load. As a result, AODV does not load any extra protocol over data packets because it doesn't use resource routing.

\subsection{DSR Protocol Definition}

A periodic routing message is not an option to be used in DSR, thus reducing the protocol load on the bandwidth of the network, saving energy, and eliminating extensive routing updates across the network. Furthermore, the two main operating models in the DSR are route search and route guard. DSR allows the node to discover the route to any destination along with multiple network tabs [13]. In DSR there is no obligation to send and receive ad messages, battery power is maintained in the mobile hosts and the host machine enters the sleep state instead of transmitting a signal. A brief advantage of DSR that the acceptance of any symmetrical connection is not required and it has a faster response to changes than distance vector based protocol.

\subsection{DSDV Protocol Definition}

It is obtained from the algorithm of Distributed Bellman-Ford (DBF). Improvements are made in the basic DBF to eliminate the loop issue. Loop formation is eliminated by labeling record of each route table with a sequence number. Packets in DSDV are transmitted among the nodes using tables of routing placed on every node. Every table of routing records all possible paths and the number of tabs to each of these destinations on every node. DSDV utilizes periodic as well as triggered routing updates to guarantee consistency of routing tables. DSDV's benefit is that it can transmit data at any moment without looping. On the other hand, DSDV has some disadvantages as well, for parameters such as maximum stability time for a specified goal; it is hard to determine ideal values. This can contribute to fake path announcements leading to path changes, and thus the bandwidth can be occupied unnecessarily. 


\section{Results}

In the evaluation, a detailed model of ns- 2 simulation was used. The wireless model utilizes a commercial wireless interface close to Lucent's wave-LAN; it is designed as a wireless communication scheme of shared media with $2 \mathrm{Mb} / \mathrm{s}$ nominal bit rate and $250 \mathrm{~m}$ nominal signal range [17]. The protocols have a packet transmit buffer equal to 64 packs.

All the packets waiting for the route response are listed, also to avoid endless buffering; packets are automatically released when they delay more than 30 seconds in the send buffer. All packets sent by the routing layer (both information and routing packets) join a queue in the interface until they are transmitted through the MAC layer [18]. The interface queue has a maximum packet size equal to 50 and is kept as a two priority queue, each running in FIFO sequence, and the routing packets take priority over data packets [19]. Constant bit rate is used for traffic sources. Source-target pairs are randomly distributed over the network [19]. A packet size of 512 bytes was used. The pair's number of source-destination and the rate at which the packets are sent in each pair depends on the load within the network. In a rectangular region, the mobility model utilizes an arbitrary pattern. The network configurations use 50 nodes in an area of $500 \mathrm{~m}^{2}$. The packet starts from a random point to a randomly selected target at a random rate (scattered within the range of 0-20 m / s) at each random journey. When the target is reached by the packet, another random target will be selected after waiting for a certain period. Waiting time that impacts mobile node relative velocities differs. For 100 seconds simulations are performed. Equivalent mobility and traffic scenarios are used in the protocols to obtain fair results [17].

\subsection{Evaluation of Results}

\subsubsection{Number of Packages Produced}

The output was calculated and plotted for AWK scripts with a standby time of $10.00 \mathrm{~s}$ for a 100 -node system over the 200 -second processing time.

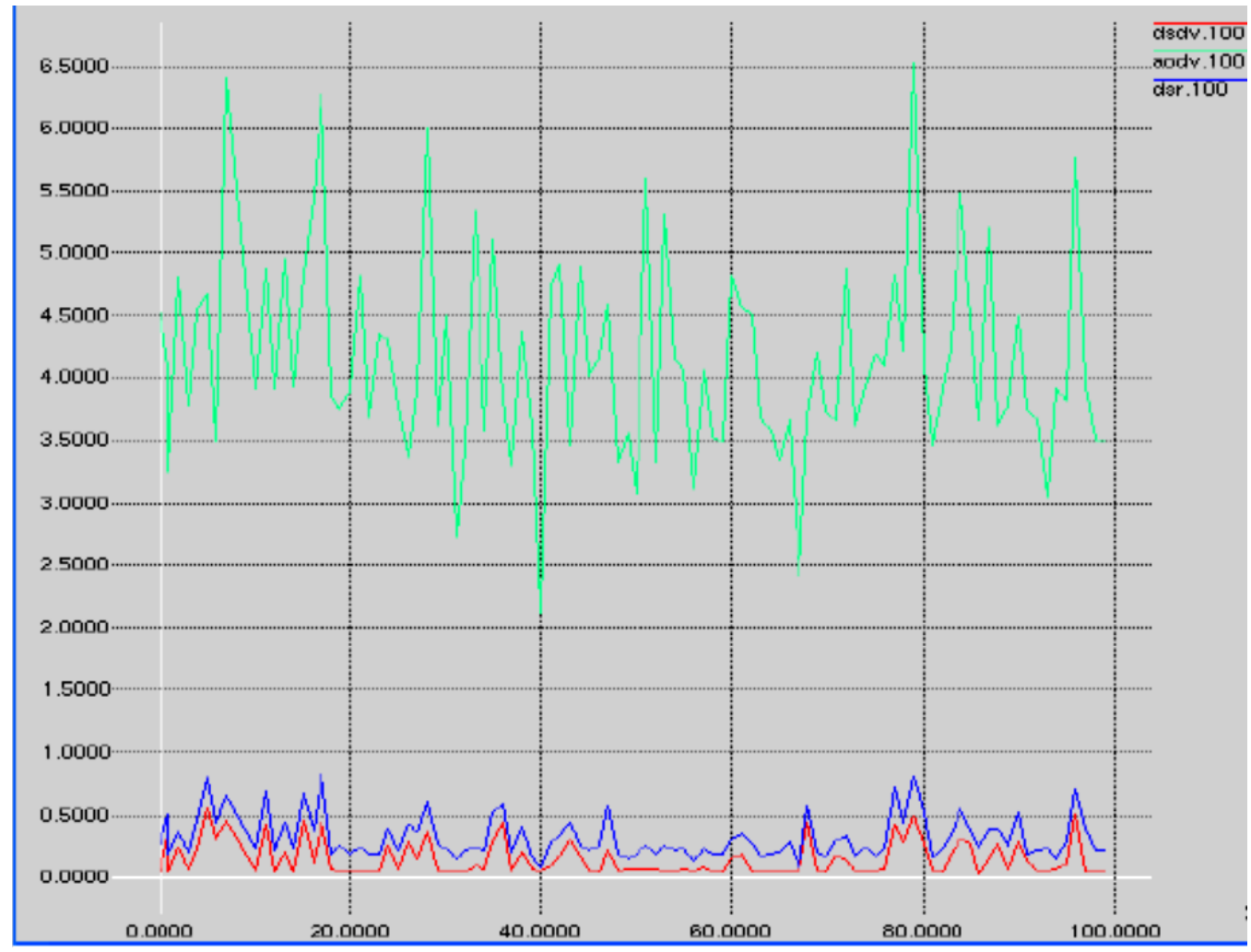

Figure 3. Number of Generated Packets 


\subsubsection{Performance comparison of protocols}

First of all, we attempted to compare all suggested protocols with similar simulation setting, the velocity of the nodes was set to $20 \mathrm{~m} / \mathrm{s}$ and the waiting time ranged from $0 \mathrm{Sec}, 10 \mathrm{Sec}, 20 \mathrm{Sec}, 40 \mathrm{Sec}$, and $100 \mathrm{Sec}$ [17]. Figure 4.A \& Figure 4.B; illustrate the performance of studied protocols. A huge number of data packets provided when there is small node mobility or in other words (large waiting period) by all protocols, while the delivery performance is close to $100 \%$ when no node movement exists. DSR \& AODV, extremely well performed by delivering approximately $85 \%$ of the data packets, Irrespective of the mobility rate.

The average delivery of end-to-end packets in DSDV was high compared to the other two protocols. In summary, both AODV and DSR exceeded DSDV performance; the reasons will be evaluated in the future. Due to the good results of both AODV protocol and DSR protocol, the efficiency variance among the two protocols was altered by changing and adjusting the mobility model and traffic sources number.

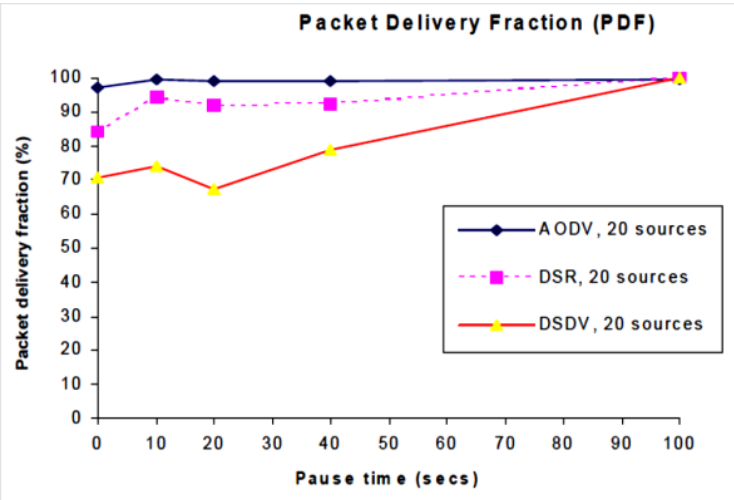

Fig. (4A)

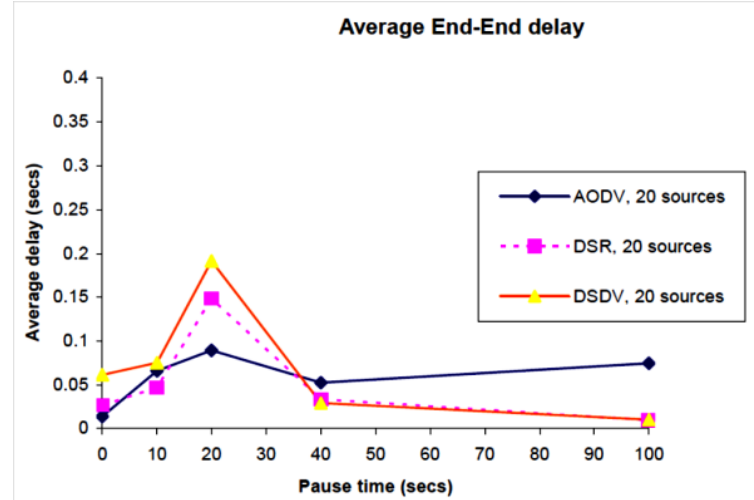

Fig. (4B)

Figure 4. PDF and Avg. end-to-end delay for 20 sources

\subsection{Changing Mobility and Resource Count}

We initiated the simulation with 40,30, 20 and 10 traffic sources. The waiting time was equal to 0 (means high mobility), and increasing step by step to be like 10, 20, 40, and at last 100 (means no mobility) and the speed of packets were 4 packs/second. DSR and AODV results were similar in 10 sources (Figure 5). However, in $40 \& 30 \& 20$ sources, AODV showed approximately $15 \%$ higher performance at lower waiting times (higher mobility) than the DSR protocol.

DSR exhibits much lower routing load compared to AODV as the sources number increases (Figure 6). Briefly, if the number of the source is low, the efficiency of DSR and AODV are close to each other regardless of mobility [17]. AODV performs better than DSR for elevated mobility situations as the number of sources rises. Data from various sources indicate that AODV is beginning to outperform DSR performance at low routing load in a high number of node environments. The route answers are a major part of the DSR routing load when the main route to the AODV's routing load comes from the route requests [17]. Moreover, AODV has more demands for routes than DSR and vice versa. 


\subsection{Observations:}

Simulation results show some important routing protocol differences. The existence of increased mobility will lead to link errors, and also during errors availability, each routing protocol responds differently. Differences between these protocols ' basic working mechanisms contribute to variations in efficiency. The DSDV has routing problems under low waiting times. Whereas DSDV at higher mobility rates (lower waiting times) shows weak performance with and the packet delivery rate drops to $70 \%$.
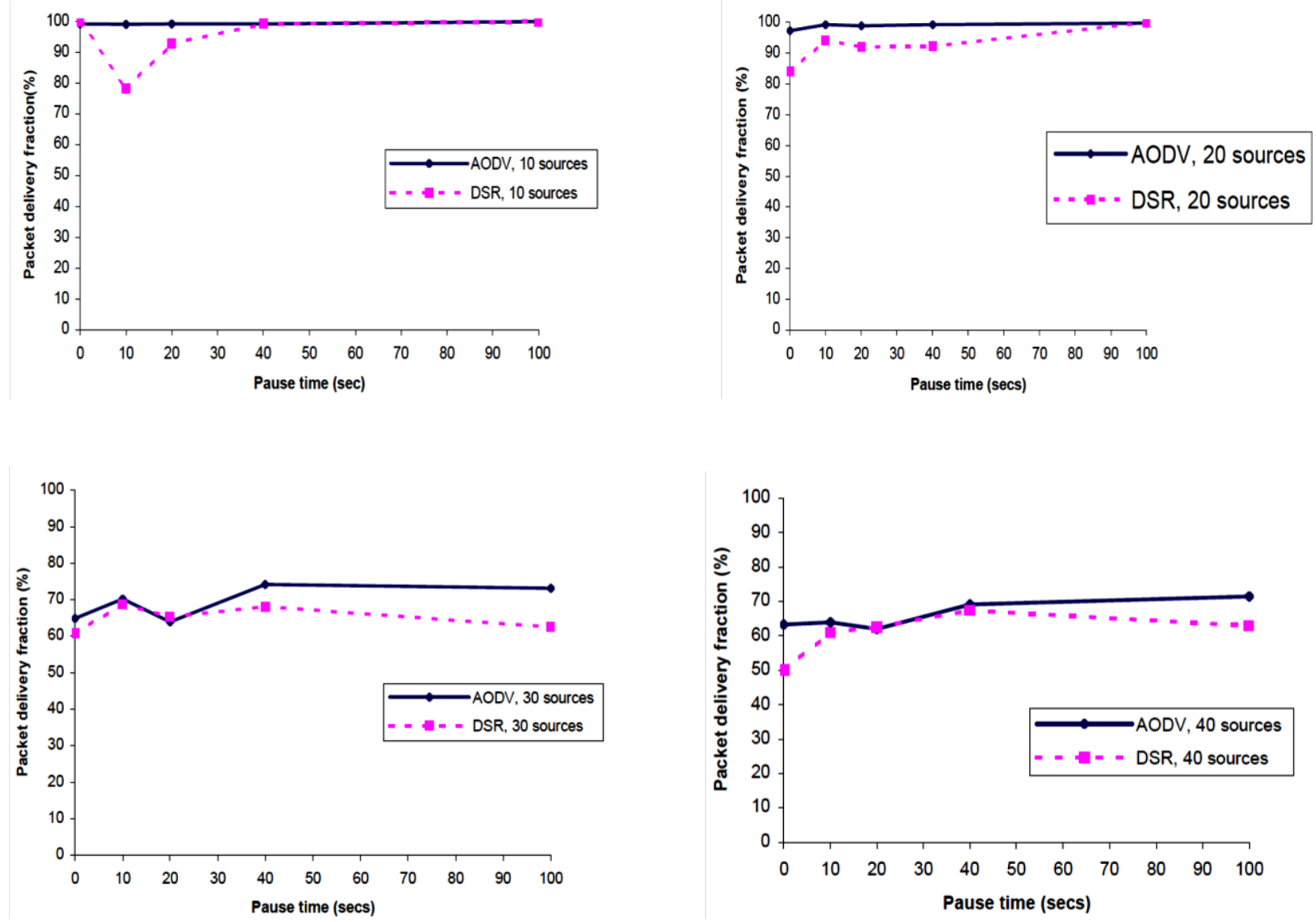

Figure 5. PDF with different number-of-sources

Nearly all of the transmitted packets were wasted because they were redirected to a broken link by an incorrect routing table record. It holds just one route for each target; therefore, any packet that the MAC layer cannot deliver is removed as no alternative path exists. The packet delivery speed for DSR and AODV was independent of the traffic load provided and in all instances; both methods provided packets ranging from $85 \%$ to $100 \%$. DSDV is a table-based protocol that cannot be adapted to high mobility path modifications. On the contrary, AODV and DSR have improved performance since routing information is generated only when needed. 

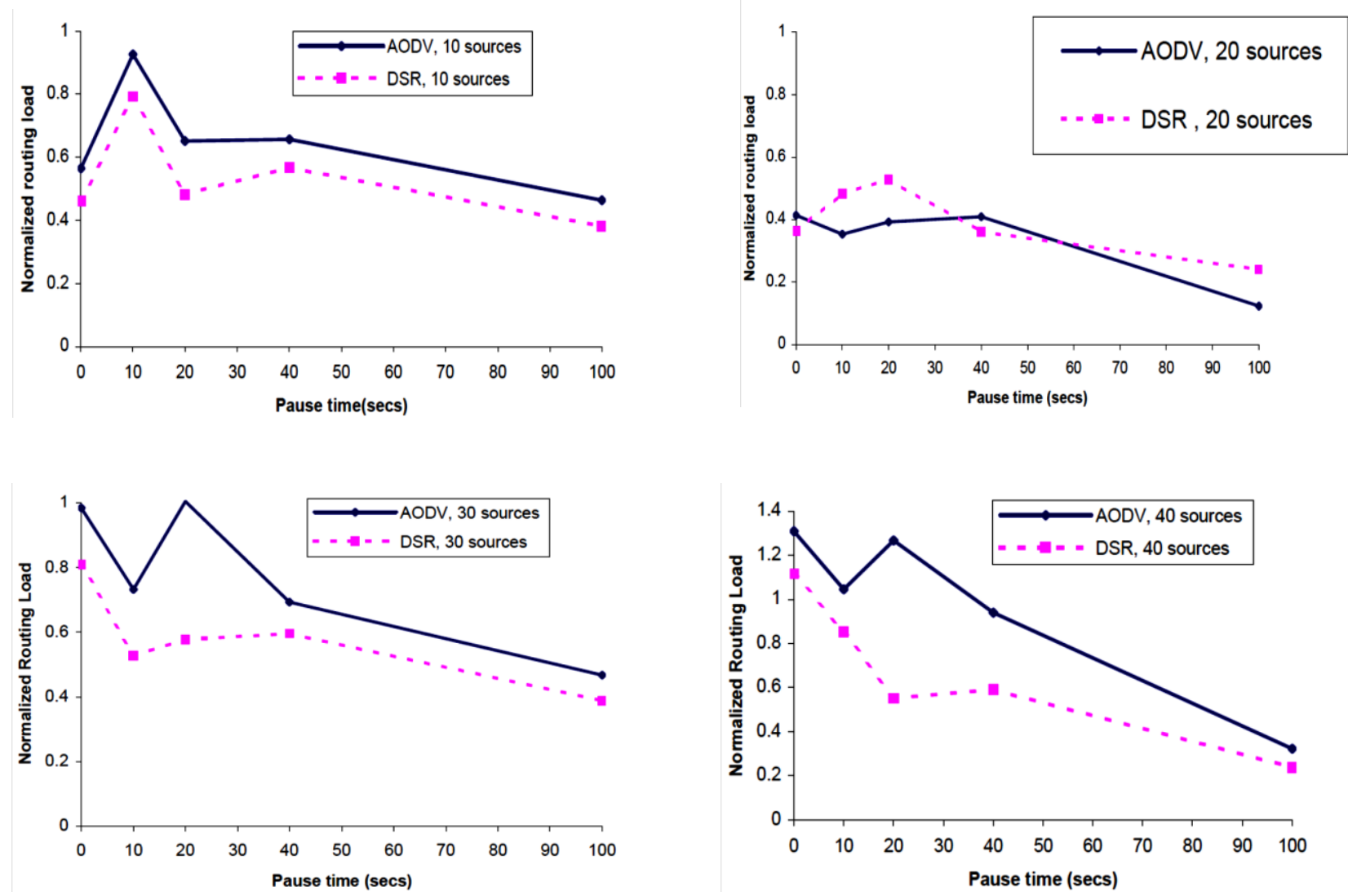

Figure (6) Normalized-Routing-Load with different number-of-sources

\section{Conclusions}

The article compared three routing protocols (DSDV, AODV, DSR) using NS2 to evaluate and measure efficiency. AODV and DSR generally, perform better in fast mobility simulations than the DSDV protocol. Fast mobility often leads to connection errors. The AODV and DSR use a demand-based search procedure for a route, but the routing mechanisms are different. The DSR uses the buffering mechanism and holds multiple paths for each location. However, for each place, AODV has one path.

According to the general observation based on the simulation, the DSR for application-based metric values, such as delay or packet delivery fraction, is used in less "stressed" environments with larger performance gaps (i.e. low node count and low load and/or mobility) shows higher performance. However, less routing load is generated by the DSR than the AODV protocol. The DSR's bad performance can be attributed primarily to the use of aggressive buffers and the lack of a method to cancel invalid links or to detect the viability of links. The aggressive buffering mechanism appears to assist the DSR with low loads and also minimizes the routing load as well. 


\section{References}

[1] M. Esmaeili, M. Abbaspour, H. Alipour, H.Mousavi. "Challenge in QoS Supporting via Integrating Differentiated Service and Multipath Routing on Mobile Ad Hoc Network", 7th IEEE International Conference on Computer and Information Technology (CIT 2007), 2007

[2] A. K. Dwivedi, Sunita Kushwaha, O. P. Vyas, "Performance of Routing Protocols for Mobile Adhoc and Wireless Sensor Networks: A Comparative Study", International Journal of Recent Trends in Engineering, Vol 2, No. 4, November 2009.

[3] Charu Wahi, Sanjay Kumar Sonbhadra, "Mobile Ad Hoc Network Routing Protocols: A Comparative Study", International Journal of Ad hoc, Sensor \& Ubiquitous Computing (IJASUC),Vol.3, No.2, April 2012.

[4] Sachin Dnyandeo Ubarhande, Performance Evolution of AODV and DSR Routing Protocols in MANET Using NS2, International Journal of Scientific \& Engineering Research, Vol. 3, Issue 5, May-2012, ISSN 2229-5518

[5] Bharathi, A Review and Comparative Study on Various Routing Protocols for MANET, International Journal of Innovative Research in Science, Engineering and Technology, Vol. 5, Issue 12, December 2016, ISSN(Online): 2319-8753

[6] B.N. Jagdale, Pragati Patil, P. Lahane, D. Javale, Analysis and Comparison of Distance Vector, DSDV and AODV Protocol of MANET, International Journal of Distributed and Parallel Systems (IJDPS), Vol.3, No.2, March 2012

[7] Md. Arafatur Rahman, Farhat Anwar, Jannatul Naeem, and Md. Sharif Minhazul Abedin, "A Simulation-Based Performance Comparison of Routing Protocol on Mobile Ad-hoc Network (Proactive, Reactive and Hybrid)", International Conference on Computer and Communication Engineering (ICCCE 2010), Kuala Lumpur, Malaysia 11-13 May 2010.

[8] Mohit Dua, Virender Ranga, Krishma Mehra, Awan Kardam, Snehal Mohan Bahsakhetre, Performance Evaluation Of Aodv, Dsr, Dsdv Mobile Ad-Hoc Protocols On Different Scenarios: An Analytical Review, International Journal Of Advances In Computing And Information Technology, February 2012, DOI: 10.6088/ijacit.12.10004, ISSN 2277-9140.

[9] S.R. Raja, K. Alagarsamy, Routing Protocols In Manet QoS Survey, International Journal of Innovative Research in Advanced Engineering (IJIRAE), Issue 5, Vol. 2, May 2015, ISSN: 23492163.

[10] Diaa Eldein Mustafa Ahmed, Othman O. Khalifa, A Comprehensive Classification of MANETs Routing Protocols, International Journal of Computer Applications Technology and Research, Issue 3, Vol. 6, 141-158, 2017, ISSN:-2319-8656.

[11] Ajay Kumar, Deepak Sharma, Sunil Kumar, Suneet Shukla, Performance Valuation Of Reactive Routing protocols, International Journal of Research in Computer Applications and Robotics, Vol.1 Issue.6, Pg: 39-44 September 2013, ISSN: 2320-7345

[12] Chander Prabha, Surender Kumar, Ravinder Khanna, Wireless Multi-hop Ad-hoc Networks: A review, IOSR Journal of Computer Engineering (IOSR-JCE), Issue 2, Vol. 16, VI (Mar-Apr. 2014), PP 54-62. e-ISSN: 2278-0661, p- ISSN: 2278-8727. 
[13] Amer O. Abu Salem, Ghassan Samara, Tareq Alhmiedat, Performance Analysis of Dynamic Source Routing Protocol, Journal of Emerging Trends in Computing and Information Sciences, Vol. 5, No. 2 February 2014, ISSN 2079-8407.

[14] Rohit Kumar, Comparative Study of Reactive and Proactive Routing Protocols, International Journal of New Innovations in Engineering and Technology, Vol. 3 Issue 3 - July 2015, ISSN: 2319-6319.

[15] Hua-Wen Tsai, Tzung-Shi Chen, Chih-Ping Chu. "An On-Demand Routing Protocol with Backtracking for Mobile Ad Hoc Networks", Wireless Personal Communications, 2006

[16] Sarkar, "Routing Protocols", Ad Hoc Mobile Wireless Networks Principles Protocols and Applications, Second Edition, 2013.

[17] Saurabh Rastogi. "Optimizing Routing Protocols for Ad Hoc Network", IEEE International Conference on Mobile Ad Hoc and Sensor Systems, 2006

[18] K Majumder, S K Sarkar, S Ray. "Analysis of QoS Parameters for DSDV and DSR in Hybrid Scenario", International Symposium on Electronic System Design, 2010

[19] Soumya Maity, Soumya K. Ghosh. "Enforcement of access control policy for mobile ad hoc networks", Proceedings of the Fifth International Conference on Security of Information and Networks - SIN '12, 2012

[20] Nariman Jabbar Qasim, et al. " Reactive protocols for unified user profiling for anomaly detection in mobile Ad Hoc networks ", Periodicals of Engineering and Natural Sciences, Vol. 7, No. 2, August 2019, pp.843-852, ISSN 2303-4521

[21] Salah A. Aliesawi, et al. " Iterative multipacket detection with FDE based MAC protocol in vehicular ad hoc networks ", Periodicals of Engineering and Natural Sciences, Vol. 7, No. 3, September 2019, pp.1041-1053, ISSN 2303-4521.

[22] Hager Ramadan, et al. " Effective data routing using mobile sinks in disjoint mobile wireless sensor networks ", Periodicals of Engineering and Natural Sciences, Vol. 7, No. 1, June 2019, pp.82-98, ISSN 2303-4521.

[23] Kavitha V, Kirupa Ganapathy. " Efficient and Optimal Routing Using Ant Colony Optimization Mechanism for Wireless Sensor Networks ", Periodicals of Engineering and Natural Sciences, Vol.6, No.1, June 2018, pp. 171 181, ISSN 2303-4521. 